\title{
Association of Changes in Physical Activity and the Incidence and Remission of Overall and Abdominal Obesity in 113,950 Adults
}

Running title: Changes in Physical Activity and Obesity

David Martinez-Gomez $\mathrm{PhD}^{1,2}$, Mark Hamer $\mathrm{PhD}^{3}$, Francisco B Ortega $\mathrm{PhD}^{4}$, Verónica Cabanas-Sanchez ${ }^{5}$, Kabir P Sadarangani MSc ${ }^{6,7}$, Carl J Lavie MD ${ }^{8}$, Fernando RodríguezArtalejo $\mathrm{MD} \mathrm{PhD}^{1,2}$.

${ }^{1}$ Department of Preventive Medicine and Public Health, Universidad Autónoma de Madrid/ IdiPaz, CIBER of Epidemiology and Public Health (CIBERESP), Madrid, Spain. ${ }^{2}$ IMDEA Food Institute. CEI UAM+CSIC, Madrid, Spain. ${ }^{3}$ School of Sport, Exercise and Health Sciences, Loughborough University, Loughborough, UK. ${ }^{4}$ PROFITH "PROmoting FITness and Health through physical activity" research group, Department of Physical Education and Sports, Faculty of Sport Sciences, University of Granada, Spain; Department of Biosciences and Nutrition at NOVUM, Karolinska Institutet, Huddinge, Sweden. ${ }^{5}$ Department of Physical Education, Sport and Human Movement, Universidad Autónoma de Madrid, Madrid, Spain.

${ }^{6}$ School of Physiotherapy, Faculty of Health Sciences, Universidad San Sebastián, Lota, Santiago, Chile. ${ }^{7}$ Escuela de Kinesiología, Facultad de Salud y Odontología, Universidad Diego Portales, Santiago, Chile. ${ }^{8}$ Department of Cardiovascular Disease, John Ochsner Heart \& Vascular Institute, Ochsner Clinical School, The University of Queensland School of Medicine, New Orleans, LA, USA.

Correspondence to: Dr. David Martinez-Gomez. Department of Preventive Medicine and Public Health, Universidad Autónoma de Madrid. E-mail: $\underline{\text { d.martinez@uam.es }}$ 


\section{* WHAT IS ALREADY KNOWN ABOUT THIS SUBJECT?}

- Most prospective cohort studies on the association between physical activity and weight change have used only a baseline measure of physical activity.

- Assessment of changes in physical activity better estimates the potential effect of physical activity-based interventions to control adiposity and provide a closer approach to causal evidence.

\section{* WHAT DOES THIS STUDY ADD?}

- In this work we found that increasing physical activity may help to prevent both overall and abdominal obesity.

- As regards to obesity management, our results also suggest that among participants with obesity, increasing physical activity may help to lose sufficient weight or reduce their waist circumference to become non-obese.

- Adopting a more active lifestyle could play a key role in the prevention and management of obesity in the population. 


\section{ABSTRACT}

Objective: We examined the dose-response relationship between changes in physical activity (PA) and subsequent incidence or remission of overall and abdominal obesity.

Methods: A total of 113,950 healthy individuals aged $\geq 18$ years participating in the Taiwan MJ Cohort. Two-year changes in PA between the first and second examination were linked to subsequent development and remission of overall and abdominal obesity.

Results: During a mean 5.6-y follow-up after the second examination, 9,991 and 11,488 individuals developed overall and abdominal obesity, respectively; also 3,588 and 3,156 participants with obesity lost sufficient weight or reduce their waist circumference to be classified as non-obese. Compared to no changes in PA, the multivariable hazard ratio (95\% confidence interval) of overall obesity was $0.95(0.90-1.00)$ for a PA increase of 0.01 to 3.74 MET-h/week, and $0.86(0.80-0.91)$ of PA increase of $\geq 3.75$ MET-h/week. Corresponding values for remission of obesity were 1.00 (0.91-1.09) and $1.16(1.05-1.28)$. Similar results were observed for the development and remission of abdominal obesity. Any decrease in PA was not associated with the risk of obesity outcomes when compared with a stable pattern (0 MET-h/week).

Conclusions: Increasing PA was consistently associated with a progressively lower incidence and higher remission of overall and abdominal obesity.

Keywords: physical activity, obesity, abdominal obesity, epidemiology. 


\section{INTRODUCTION}

Overall and abdominal obesity have reached epidemic levels worldwide and represent an enormous health (1-4) and economic burden (5-6). Physical activity (PA) promotes a negative energy balance by increasing total energy expenditure $(1,7)$ and, thus, could play a key role in both prevention and management of obesity. Accordingly, public health and obesity management guidelines highlight PA as a significant contributor to weight maintenance and weight loss, but evidence is still insufficient to inform effective populationbased strategies (8-13).

Most previous epidemiological studies on the association between PA and weight change have used only baseline measurements of PA $(13,14)$. However, the assessment of changes in PA levels could serve to estimate more accurately the potential effect of PA-based interventions to control weight and adiposity, and also provide a closer approach to causal relationships (15-18). To date, only a few studies have examined the prospective association between changes in PA and obesity. However, these studies have limitations such as the use of weight without any standardization as the main outcome, obesity measurements based on self-report, restricted use of obesity outcomes as continuous variables, inclusion of participants with any class of obesity, and no stratified analyses across obesity groups (1518). On the other hand, exercise-based clinical trials with obese adults have been of short duration (i.e. 6-12 months) and were conducted in relatively small and motivated samples, under highly structured regimes; thus, their results might not represent the long-term benefits of PA in most individuals with obesity (14).

The present study, conducted with a large prospective cohort with repeated measures of PA and objective overall and abdominal obesity in Asian adults, examined the doseresponse relationship between changes in PA and subsequent incidence and remission of obesity. 


\section{METHODS}

\section{Study population}

This research was conducted using the Taiwan MJ Cohort resource, an ongoing and dynamic prospective study of women and men who participated in a large health check-up program run by the MJ Health Management Institution, Taiwan (www.mjclinic.com.tw). The details of the MJ Cohort study population and data collection methods have been reported elsewhere $(19,20)$. Briefly, the MJ Cohort has enrolled around 600,000 Taiwanese individuals since 1994. Participants completed a standardized protocol including a self-administered questionnaire, physical examinations, and laboratory tests in the morning; these procedures conformed to ISO 9001 for quality management. After the first examination, all participants were encouraged to return annually, and all data are updated by the MJ Health Research Foundation.

Study participants provided informed written consent, and the study protocol was approved by the Institutional Review Boards of the MJ Health Management Institution and the National Health Research Institutes in Taiwan. Individual identification data were removed, so that participants remained anonymous during the entire study process (21). To avoid research inaccuracies caused by the quality of the data, the MJ Health Research Foundation previously conducted standardized data cleaning (e.g. extreme or incorrect values) (22).

For this analysis, we selected men and women aged $\geq 18$ years who attended the first examination (baseline) during 1997-2016. The exclusion criteria were: (i) history of cancer or cardiovascular disease (CVD) at the first and/or second examinations, (ii) incomplete data at the first examination on main covariates, with the exception of educational attainment and marital status, which were replaced with information from the next examination with these data, (iii) having less than three examinations through 2016 with valid data on the specific 
obesity variables, (iv) lacking data at the first and second examinations on PA data, and (v) incidence or remission of obesity at the second examination.

\section{Study variables}

The time spent in leisure-time PA have been assessed by the MJ PA Questionnaire since $1997(23,24)$. In this questionnaire participants were asked to report participation in the intensity, frequency, and duration of PA during the last 4 weeks, with several examples of activity types given for four intensity categories: light (e.g. slow walking), moderate (e.g. brisk walking), medium-vigorous (e.g. jogging), or high-vigorous (e.g. running). A metabolic equivalent (MET; $3.5 \mathrm{ml} / \mathrm{kg} / \mathrm{min}$ ) value of 2.5 was assigned for light, 4.5 for moderate, 6.5 for medium-vigorous, and 8.5 for high-vigorous PA intensity $(23,24)$. To calculate the total volume of PA (MET-h/week), the MET value for the reported intensity was multiplied by the frequency and duration. For participants who indicated activities in more than one intensity category, an average MET value was assigned. The MJ PA Questionnaire has been slightly modified over the years and all the data were harmonized before pooling (Supplementary Table 1) as detailed previously (24). This questionnaire has shown acceptable reliability and excellent convergent validity predicting cause-specific mortality and cancer incidence (23). Change in PA as a continuous variable was calculated as the difference in total volume of PA at the first and second examinations, and divided by number of years between them (MET$\mathrm{h}$ /week per year) because the interval between examinations varied among participants in this cohort. Participants with an annual change of 0 MET-h/week were categorized into the stable or no change group, whereas those with negative and positive values were categorized into the decrease and increase groups, respectively. Among these latter groups, the cut-off point of 3.75 MET-h/week per year (equivalent to $1 / 2$ times the minimum recommended amount of PA, that is, 75 min per week of moderate intensity PA or $37.5 \mathrm{~min}$ at vigorous intensity) was used 
to define small to moderate vs. moderate to large changes in PA; this amount of PA has been previously associated with a reduced risk of obesity (24).

From the first to the last examination, body weight to the nearest $0.1 \mathrm{~kg}$ and height to the nearest mm were measured using an automatic anthropometer (Nakamura KN-5000A, Tokyo, Japan). Also, waist circumference (WC) to the nearest mm was measured at the midpoint between the lower end of the rib cage and the crest of the ilium. BMI was calculated as weight in $\mathrm{kg}$ divided by squared height in $\mathrm{m}$, and participants with values $\geq 25 \mathrm{~kg} / \mathrm{m}^{2}$ were classified as obese according to guidelines for Asian populations (25). Asian cut-off points of $\mathrm{WC} \geq 90 \mathrm{~cm}$ in men and $\geq 80 \mathrm{~cm}$ in women identified participants with abdominal obesity (26).

In the clinical examinations, participants also reported their educational attainment (middle school or below, high school, junior school, college or above), and marital status (single, married, widowed). Information on smoking and alcohol consumption (never, former, currently) based on standard questions was recorded at the first and second examinations. An optimal meal pattern at the first and second examinations was defined as an affirmative answer to the following question: "Do you eat on time and in regular amounts?" (24). Hypertension was identified from the medical history, systolic blood pressure $\geq 140 \mathrm{~mm} \mathrm{Hg}$ or diastolic blood pressure $\geq 90 \mathrm{~mm} \mathrm{Hg}$. Hypercholesterolemia was defined as serum total cholesterol $\geq 240 \mathrm{mg} / \mathrm{dL}$ or use of lipid-lowering drugs. Type 2 diabetes was defined as a history of diabetes or fasting blood glucose $\geq 126 \mathrm{mg} / \mathrm{dL}$.

\section{Statistical analyses}

Baseline characteristics of the total sample and by study groups were summarized as mean and SD for continuous variables, and as percentage for categorical variables. Follow-up time was computed as the difference between the date of the second examination and the date 
of the examination where overall and abdominal obesity was developed or remitted for the first time or the last examination through the end of 2016.

To model the dose-response relationship between changes in PA and overall and abdominal obesity risk and remission, we used restricted cubic spline Cox regression with knots at the $10^{\text {th }}, 50^{\text {th }}$, and $90^{\text {th }}$ percentile, and a change of 0 MET-h/week as the reference value; these knots ensure that the curve interpolates the end points assuming a Gaussian distribution. Departure from linearity was assessed by a Wald test and an F statistic is computed to calculate the significance level of the hypothesis test. Total PA in MET-h/week per year was previously truncated (winsorization) at the $<1$ st and $>99$ th percentile to minimize the influence of outliers. Also Cox regression was used to estimate hazard ratios (HRs) and their 95\% confidence interval (CI) for obesity incidence and remission across the following categories of change in PA: moderate to large decrease ( $\leq-3.75$ MET-h/week), small to moderate decrease (-0.01 to $-3.74 \mathrm{MET}-\mathrm{h} / \mathrm{week})$, no change or stable, small to moderate increase (0.01 to $3.74 \mathrm{MET}-\mathrm{h} /$ week), and moderate to large increase ( $\geq 3.75$ METh/week); no change was the reference category. We built three Cox models with sequential adjustment for potential confounders: model 1 adjusted for age (years), sex (men, women), year of baseline examination (year), educational attainment (middle school or below, high school, junior school, college or above), marital status (single, married), BMI ( $\mathrm{kg} / \mathrm{m}^{2}$ ) and PA at baseline (MET-h/week); model 2 further adjusted for hypertension (yes, no), hypercholesterolemia (yes, no) and diabetes (yes, no); and model 3 further adjusted for baseline and changes in smoking (never to never, never to current, past to current, current to past, and current to current, never/past/current to missing), alcohol drinking (never to never, never to current, past to current, current to past, and current to current, never/past/current missing), and meal pattern (optimal to optimal, optimal to suboptimal, suboptimal to optimal, 
suboptimal to suboptimal, never/past/current to missing). WC $(\mathrm{cm})$ at baseline was also used as a covariate in abdominal obesity analyses.

Since severe obesity (BMI $>30 \mathrm{~kg} / \mathrm{m}^{2}$ in Asian populations) (21) has a greater harmful effect on health, we also examined the effect of changes in PA on severe obesity incidence and remission. In addition, to minimize reverse causation, we repeated the analyses among participants without baseline metabolic disturbances (hypertension, hypercholesterolemia and diabetes).

Finally, to further examine the effect of baseline PA levels on the association between changes in PA on overall and abdominal obesity risk and remission, we categorized participants in the first and second examinations as inactive (0 MET-h/week), insufficiently active ( $>0$ to $7.49 \mathrm{MET}-\mathrm{h} /$ week), and active ( $\geq 7.5 \mathrm{MET}-\mathrm{h} /$ week [equivalent to 1 time the minimum recommended amount of PA], thus creating nine groups. Cox regressions were run using participants who were inactive at baseline and follow up as the reference category, with adjustment for the aforementioned covariates plus the number of years between both examinations.

The proportional hazards assumption was checked by assessing the log-log survival plots. Statistical significance was set at 2 -sided $P<0.05$. Analyses were performed with STATA v.14. 


\section{RESULTS}

According to the exclusion criteria, a total of 113,950 individuals with the first examination between 1997-2013 were included for this work. Specifically, our final sample included 83,567 and 91,700 participants without overall and abdominal obesity at the first and second examinations, respectively, for obesity risk analyses. In addition, we included 24,153 and 12,688 participants with overall and abdominal obesity at the first and second examinations, respectively, for obesity remission analyses. Figure 1 shows the flow of participants for the present analysis of the Taiwan MJ Cohort. At baseline, study participants had a mean \pm SD age of $37.8 \pm 11.4$ years, $49 \%$ were women, $66 \%$ reported junior school or university studies, 66\% were married, and were mostly never smokers and drinkers (Table 1). Prevalence of hypertension, hypercholesterolemia, and type 2 diabetes was $13 \%, 10 \%$, and $3 \%$, respectively. Men, older participants, those with lower educational attainment, married, and with a worse metabolic profile were more likely to have overall or abdominal obesity (Table 1).

On average, the interval between the first and second examinations was $1.9 \pm 1.6$ years with an annual change in PA levels of 0.5 $\pm 5.8 \mathrm{MET}-\mathrm{h} /$ week. Participants with obesity had a slightly greater increase in PA between the first and second examinations than their counterparts without obesity (Supplementary Figure 1). Baseline characteristics according to categories of change in PA among participants with and without obesity are shown in Supplementary Tables 2-5. A total of 9,991 of 83,567 and 11,488 of 91,700 participants developed overall and abdominal obesity, respectively, during a mean \pm SD follow-up of 5.7 \pm 4.1 and 5.6 \pm 4.1 years; the corresponding incidence rates were 20.9 and 22.3 per 1,000 person-years. During a mean follow-up of 5.0 \pm 3.8 and $4.4 \pm 3.5$ years, 3,588 of 24,153 and 3,156 of 12,688 obese participants lost sufficient weight or WC to be classified as non-obese, respectively; the corresponding remission rates were 29.7 and 56.5 per 1,000 person-years. 
Restrictive cubic spline models (Figure 2) showed a non-linear dose-response association between changes in PA and subsequent incidence and remission of overall and abdominal obesity (all $P$ for non-linear trend $<0.001$ ). We observed a reduced incidence of obesity (Figures 2A and 2B) and an increased obesity remission (Figures 2C and 2D) among participants who increased PA during follow-up; and the greater the increase in PA, the stronger the favorable association with incidence of obesity or its remission. In contrast, decreasing PA levels did not show a clear association with obesity outcomes, but we found some tendency to increased obesity remission (Figures $2 \mathrm{C}$ and 2D) among those who decreased PA (a U-shaped dose-response curve).

As compared with having stable PA levels, a moderate to large increase in PA was associated with $14 \%$ and $16 \%$ lower incidence of subsequent overall and abdominal obesity, respectively (Table 2); even a small to moderate increase in PA showed a 5\% lower risk of overall obesity and a $6 \%$ lower risk of abdominal obesity. Moreover, a moderate to large increase in PA was linked to a $16 \%$ and $22 \%$ higher frequency of remission of overall and abdominal obesity, respectively (Table 2). A decrease in PA was not significantly associated with either a greater obesity development or remission (Table 2). In these models, baseline PA was significantly associated with obesity incidences, but not with obesity remissions.

Similar associations were found when examining severe obesity incidence and remission (Supplementary Figure 2 and Table 6). When excluding participants with an unfavorable metabolic profile, the results were virtually identical, with the exception of a $20 \%$ higher remission of abdominal obesity in participants with a moderate to large decrease in PA compared to those with stable PA (Supplementary Table 7).

Inactive participants at the first examination who increased PA in the second examination showed lower incidence of both types of obesity and higher frequency of remission, as compared with consistently inactive participants (Figure 3). Also, those who 
were consistently active over time had a notable reduction of incident overall and abdominal obesity (Figure 3). Participants who were initially active but became inactive in the second examination had a lower frequency of overall obesity reduction that those who were continually inactive (Figure 3). 


\section{DISCUSSION}

In this large prospective cohort of Asian men and women, increasing PA was associated with a progressively lower incidence and higher remission of overall and abdominal obesity, irrespective of some important potential confounders, including baseline PA. These findings have public health and clinical relevance by highlighting PA promotion could be an important strategy with potential to significantly curve the obesity epidemic in the general population. $(11,12)$

People with obesity are at increased risk of many serious chronic conditions, including hypertension, dyslipidemia, type 2 diabetes, CVD and cancer; and it also translates in higher mortality $(2,4)$. Thus, from a public health perspective, avoiding obesity development and its progression is crucial for future health. Increasing PA or maintaining high levels of PA have been included as key goals in public health guidelines for obesity prevention $(10,11)$. Evidence for these recommendations are mainly based on observational studies rather than clinical trials (13). The majority of these observational studies, however, were conducted with only a single measure of PA at baseline $(12,13)$. Regarding a few studies that have examined changes or trajectories in PA, weight, WC or other obesity outcomes were analyzed as continuous variables, and therefore, participants with any class of obesity were included. For example, in 3554 men and women (age 18- 30 years at baseline) from the Coronary Artery Risk Development in Young Adults (CARDIA) study, Hankinson et al. (15) evaluated the relationship between PA and changes in BMI and WC over 20 years. May et al. examined the effect of change in PA on body fatness over a 10-y period in 4944 participants from the Doetinchem Study (age 26-66 years at baseline). Hamer et al. (17) examined PA patterns over 10 years in relation to BMI and WC in 4,880 participants (mean age 49.3 years at baseline) from the Whitehall II cohort. Lee et al. (18) examined the association of different amounts of PA with long-term weight changes among women consuming a usual diet, involving 34,079 
healthy US women (mean age, 54.2 years at baseline) from 1992-2007. All these studies found a negative relationship between changes in PA and gain in overall and abdominal obesity markers.

Our previous study with baseline data on PA among 200.000 participants in this same cohort showed that, as compared with inactive participants (i.e. 0 MET-h/week), those who spent $0.1-3.74,3.75-7.49,7.50-14.99$, and $\geq 15$ MET-h/week in PA reduced their obesity risk by $2 \%, 8 \%, 10 \%$ and $10 \%$, respectively. Although previous research and current PA guidelines suggest a minimum of 7.5 MET-h/week (i.e. 150 min per week of moderate intensity PA or 75 min at vigorous intensity) for obesity prevention, this initial work showed that doing at least $1 / 2$ of the recommended amount of PA (i.e. 3.75 MET-h/week) may prevent the onset of overall obesity (20). The present study extends knowledge by confirming that increasing such amounts of PA may have certain benefits preventing obesity development: persons without obesity who increased PA at least 3.75 MET-h/week per year reduced the risk of overall obesity by $14 \%$ when compared to those with stable PA, irrespective of their baseline PA. In contrast to prior work (20), our study indicates that more benefits may be attained with a greater increase in PA (>3.75 MET-h/week per year), even though only a very small percentage of participants achieved such increase. In addition, our results also support that maintaining high PA levels would be important for obesity prevention.

Curving the obesity epidemic requires a focus on prevention, but effective management of obesity is also important. Anti-obesity medications and bariatric surgery are recommended in severe or complicated obesity, but the first-line treatment for obesity is lifestyle modification, including increased PA $(9,11,12)$. In the absence of dietary restriction, the minimum levels of PA recommended may induce only a modest weight loss (i.e. $2-3 \mathrm{~kg}$ ); thus, at least 1.5 times the recommended amount of PA (i.e. $\geq 11.25$ MET-h/week) is indicated in accordance with clinical trials without changes in dietary intake among patients with 
obesity $(11,14)$. This large cohort study has allowed to examine how participants with obesity moved to a healthier non-obese status, as well as the role of changes in PA on obesity remission during a longer follow-up than most clinical trials. In contrast to prior trial evidence, an increase of at least $1 / 2$ times the usually recommended amount of PA was associated with a $16 \%$ higher frequency of obesity remission, compared with no change PA during follow-up. Also, our results suggest that only increasing PA, but not initial PA levels or maintaining high PA, is essential for obesity remission.

We did not obtain clear results on the association between decreased PA and obesity outcomes. While analyses using categories of change in PA and adjusting for baseline PA did not show that decreased PA was linked to higher incidence of obesity (Table 2), analyses using a continuous variable of change in PA (cubic splines in Figure 2) suggest some tendency for higher risk of obesity. However, when analyses where stratified according to PA at baseline (Figure 3), individuals who were active o insufficiently active at first examination and that decreased their PA at the second examination experienced a lower reduction in obesity risk than those who remained active in both examinations. Likewise, those who were initially active but became inactive in the second examination had a lower frequency of obesity reduction that those continually active. Taking all these results together, it seems that some, but not all, of the benefits of PA remain in those who reduce their activity level. In fact, previous research has shown that the health benefits of doing high and very high levels of PA remain during some time $(13,14)$; moreover, PA is an important determinant of cardiorespiratory fitness, which is a more stable biological marker with a strong inverse relationship with obesity $(2,13)$ and critical component for predicting major clinical outcomes in obesity $(2,11,27)$. Future research with a longer follow-up may shed more light in this issue; for instance, one study on the timing of dietary changes on subsequent health outcomes 
indicates that the associations were strengthened when changes over a longer duration were evaluated (28).

Another important feature of this study was the assessment of abdominal obesity, because most previous research focused on BMI-based overall obesity $(13,14)$ and there is evidence that abdominal obesity is a risk factor for CVD and death independently of BMI (29). Our results agree with data from prospective studies and clinical trials since the beneficial effect of PA is usually greater for abdominal than for overall obesity (14). In addition, since our results are independent of BMI levels, it supports the contention that people exercising regularly may reduce abdominal fat, as measured by waist circumference, regardless of weight change (14).

The strengths of this study include a large sample size, a wide age range, objective measures of obesity, and repeated assessments of exposure and outcome variables. However, several limitations should be acknowledged. PA was self-reported, which usually overestimates PA levels and add a source of error and inaccuracy to the PA measurement. Self-reported PA data might underestimate the true effect of the associations and hence, more accuracy measurements would be important. However, a few prospective studies that used objectively measured PA, with smaller sample sizes (i.e. 47-739 participants) and shorter lengths of follow-up (i.e. 1.5-5.6 years) than reports with self-reported PA, found mixed findings on the association between baseline and changes in PA and different obesity markers (30). Also, PA was limited to leisure-time domains; thus, the dose-response association of changes in PA at different intensities in other domains (i.e., occupational, household and transportation) with incidence and remission of obesity are of major importance and should still be investigated (31-33). Lack of information on other potentially important variables (e.g. energy intake, medications, family history of CVD) is also a major limitation in the present work. Another limitation is that participants may not be representative of the general 
population of Taiwan, because they had somewhat higher socioeconomic status; however, the prevalence of obesity and other characteristics in this cohort are similar to that in the Taiwanese population (19). Also, these analyses should be replicated in other Asian and nonAsian cohorts because the results may differ. Finally, although analyses were adjusted for many variables, including changes in lifestyle over time, some residual confounding cannot be ruled out.

In conclusion, in this large Asian cohort, even a relatively small increase in PA was associated with a lower incidence, as well as higher remission, of overall and abdominal obesity. These results suggest that adopting a more active lifestyle could play a key role in prevention and management of obesity in the population with potentially profound public health implications. Future research should confirm our results in other populations, and evaluate the effectiveness of interventions to better promote PA at the individual and population levels (12). 


\section{ACKNOWLEDGMENTS}

We thank MJ Health Research Foundation for making their large dataset available to us.

\section{AUTHOR CONTRIBUTIONS}

DMG and FRA had full access to all of the data in the study and take responsibility for the integrity of the data and the accuracy of the data analysis. Study concept and design: DMG, FBO, MH, and CJL. Analysis and interpretation of data: all authors. Drafting of the manuscript: DMG, FBO, MH, CJL, and FRA. Critical revision of the manuscript for important intellectual content: FBO, MH, VCS, KPS, and CJL. Statistical analysis: DMG, FBO, and MH. Administrative, technical, or material support: VCS, KPS. Study supervision: FRA. All authors have read and approved the final manuscript.

\section{FUNDING}

This work was supported by Grant 02/2014 from the Plan Nacional sobre Drogas (Ministry of Health of Spain), FIS grants 12/1166 and 16/609 (State Secretary of R+D+I and FEDER/FSE), and MINECO R+D+I grant (DEP2013-47786-R). The funders had no role in study design, data collection and analysis, decision to publish, or preparation of the manuscript. The MJ cohort was supported by the MJ Health Management Institution. All data used in this research were authorized by, and received from MJ Health Research Foundation by MJ Health Research Center (Authorization Codes: MJHRF2017006A, MJHRF2017007A and MJHRF2018004A). Any interpretation or conclusion achieved in this work does not represent the views of MJ Health Research Foundation. The Taiwan MJ Cohort is available for external researchers. Applicants for data access should contact the MJ Health Research Foundation at www.mjhrf.org.

\section{COMPETING INTERESTS}

All authors declare that there are no conflicts of interest. 


\section{REFERENCES}

1. González-Muniesa P, Mártinez-González MA, Hu FB, Després JP, Matsuzawa Y, Loos RJF, Moreno LA, Bray GA, Martinez JA. Obesity. Nat Rev Dis Primers. 2017 Jun $15 ; 3: 17034$

2. Ortega FB, Lavie CJ, Blair SN. Obesity and Cardiovascular Disease. Circ Res. 2016;118(11):1752-70.

3. Kelly T, Yang W, Chen CS, Reynolds K, He J. Global burden of obesity in 2005 and projections to 2030. Int J Obes (Lond). 2008;32(9):1431-7.

4. Heymsfield SB, Wadden TA. Mechanisms, Pathophysiology, and Management of Obesity. N Engl J Med. 2017;376(3):254-266.

5. Withrow D, Alter DA. The economic burden of obesity worldwide: a systematic review of the direct costs of obesity. Obes Rev. 2011;12(2):131-41.

6. Wang YC, McPherson K, Marsh T, Gortmaker SL, Brown M. Health and economic burden of the projected obesity trends in the USA and the UK. Lancet. 2011;378(9793):815-25.

7. Hill JO, Wyatt HR, Peters JC. Energy balance and obesity. Circulation. 2012;126(1):126-32.

8. Yumuk V, Tsigos C, Fried M, Schindler K, Busetto L, Micic D, Toplak H; Obesity Management Task Force of the European Association for the Study of Obesity. European Guidelines for Obesity Management in Adults. Obes Facts. 2015;8(6):40224.

9. Jensen MD, Ryan DH, Apovian CM, Ard JD, Comuzzie AG, Donato KA, Hu FB, Hubbard VS, Jakicic JM, Kushner RF, Loria CM, Millen BE, Nonas CA, Pi-Sunyer FX, Stevens J, Stevens VJ, Wadden TA, Wolfe BM, Yanovski SZ, Jordan HS, Kendall KA, Lux LJ, Mentor-Marcel R, Morgan LC, Trisolini MG, Wnek J, Anderson JL, 
Halperin JL, Albert NM, Bozkurt B, Brindis RG, Curtis LH, DeMets D, Hochman JS, Kovacs RJ, Ohman EM, Pressler SJ, Sellke FW, Shen WK, Smith SC Jr, Tomaselli GF; American College of Cardiology/American Heart Association Task Force on Practice Guidelines; Obesity Society. 2013 AHA/ACC/TOS guideline for the management of overweight and obesity in adults: a report of the American College of Cardiology/American Heart Association Task Force on Practice Guidelines and The Obesity Society. Circulation. 2014 Jun 24;129(25 Suppl 2):S102-38.

10. Kumanyika SK, Obarzanek E, Stettler N, Bell R, Field AE, Fortmann SP, Franklin BA, Gillman MW, Lewis CE, Poston WC 2nd, Stevens J, Hong Y; American Heart Association Council on Epidemiology and Prevention, Interdisciplinary Committee for Prevention. Population-based prevention of obesity: the need for comprehensive promotion of healthful eating, physical activity, and energy balance: a scientific statement from American Heart Association Council on Epidemiology and Prevention, Interdisciplinary Committee for Prevention (formerly the expert panel on population and prevention science). Circulation. 2008 Jul 22;118(4):428-64.

11. Lavie CJ, Laddu D, Arena R, Ortega FB, Alpert MA, Kushner RF. Healthy Weight and Obesity Prevention: JACC Health Promotion Series. J Am Coll Cardiol. 2018;72(13):1506-1531

12. Fletcher GF, Landolfo C, Niebauer J, Ozemek C, Arena R, Lavie CJ. Promoting Physical Activity and Exercise: JACC Health Promotion Series. J Am Coll Cardiol. 2018;72(14):1622-1639.

13. 2018 Physical Activity Guidelines Advisory Committee. 2018 Physical Activity Guidelines Advisory Committee Scientific Report. Washington, DC: U.S. Department of Health and Human Services, 2018. 
14. Swift DL, McGee JE, Earnest CP, Carlisle E, Nygard M, Johannsen NM. The Effects of Exercise and Physical Activity on Weight Loss and Maintenance. Prog Cardiovasc Dis. 2018;61(2):206-213.

15. Hankinson AL, Daviglus ML, Bouchard C, Carnethon M, Lewis CE, Schreiner PJ, Liu K, Sidney S. Maintaining a high physical activity level over 20 years and weight gain. JAMA. 2010;304(23):2603-10.

16. May AM, Bueno-de-Mesquita HB, Boshuizen H, Spijkerman AM, Peeters PH, Verschuren WM. Effect of change in physical activity on body fatness over a 10-y period in the Doetinchem Cohort Study. Am J Clin Nutr. 2010;92(3):491-9.

17. Hamer M, Brunner EJ, Bell J, Batty GD, Shipley M, Akbaraly T, Singh-Manoux A, Kivimaki M. Physical activity patterns over 10 years in relation to body mass index and waist circumference: the Whitehall II cohort study. Obesity (Silver Spring). 2013;21(12):E755-61.

18. Lee IM, Djoussé L, Sesso HD, Wang L, Buring JE. Physical activity and weight gain prevention. JAMA. 2010 Mar 24;303(12):1173-9.

19. Wu X, Tsai SP, Tsao CK, Chiu ML, Tsai MK, Lu PJ, Lee JH, Chen CH, Wen C, Chang SS, Hsu CY, Wen CP. Cohort Profile: The Taiwan MJ Cohort: half a million Chinese with repeated health surveillance data. Int J Epidemiol. 2017. 46(6):17441744.

20. MJ Health Research Foundation. The introduction of MJ Health Database. MJ Health Research Foundation, Technical Report MJHRF-TR-01, 2016.

21. Chuang YC. MJ Health data-cleaning procedure. MJ Health Research Foundation, Technical Report MJHRF-TR-04, 2016.

22. Chuang YC. MJ Health Data information security management guidelines. MJ Health Research Foundation, Technical Report MJHRF-TR-03, 2016. 
23. Wen CP, Wai JP, Tsai MK, Yang YC, Cheng TY, Lee MC, Chan HT, Tsao CK, Tsai SP, Wu X. Minimum amount of physical activity for reduced mortality and extended life expectancy: a prospective cohort study. Lancet. 2011;378(9798):1244-53.

24. Martinez-Gomez D, Esteban-Cornejo I, Lopez-Garcia E, Garcia-Esquinas E, Sadarangani KP, Veiga OL, Rodríguez-Artalejo F. Physical activity less than the recommended amount may prevent the onset of major biological risk factors for cardiovascular disease: A cohort study of 198,919 adults. Br J Sport Med. In press.

25. World Health Organization. The Asia-Pacific perspective: redefining obesity and its treatment. Sydney, Australia: Health Communications Australia Pty Limited; 2000.

26. Alberti KG, Eckel RH, Grundy SM, Zimmet PZ, Cleeman JI, Donato KA, Fruchart JC, James WP, Loria CM, Smith SC Jr; International Diabetes Federation Task Force on Epidemiology and Prevention; Hational Heart, Lung, and Blood Institute; American Heart Association; World Heart Federation; International Atherosclerosis Society; International Association for the Study of Obesity. Harmonizing the metabolic syndrome: a joint interim statement of the InternationalDiabetes Federation Task Force on Epidemiology and Prevention; National Heart, Lung, and Blood Institute; American Heart Association; World Heart Federation; International Atherosclerosis Society; and International Association for the Study of Obesity. Circulation. 2009; 120: 1640-5.

27. Kennedy AB, Lavie CJ, Blair SN. Fitness or Fatness: Which Is More Important? JAMA. 2018;319(3):231-232.

28. Sotos-Prieto M, Bhupathiraju SN, Mattei J, Fung TT, Li Y, Pan A, Willett WC, Rimm EB, Hu FB. Association of Changes in Diet Quality with Total and Cause-Specific Mortality. N Engl J Med. 2017 Jul 13;377(2):143-153. 
29. Hoffmann K, Bergmann M, Schulze MB, Overvad K, van der Schouw YT, Spencer E, Moons KG, Tjønneland A, Halkjaer J, Jensen MK, Stegger J, Clavel-Chapelon F, Boutron-Ruault MC, Chajes V, Linseisen J, Kaaks R, Trichopoulou A, Trichopoulos D, Bamia C, Sieri S, Palli D, Tumino R, Vineis P, Panico S, Peeters PH, May AM, Bueno-de-Mesquita HB, van Duijnhoven FJ, Hallmans G, Weinehall L, Manjer J, Hedblad B, Lund E, Agudo A, Arriola L, Barricarte A, Navarro C, Martinez C, Quirós JR, Key T, Bingham S, Khaw KT, Boffetta P, Jenab M, Ferrari P, Riboli E. General and abdominal adiposity and risk of death in Europe. N Engl J Med. 2008;359:210520.

30. Wilks DC, Besson H, Lindroos AK, Ekelund U. Objectively measured physical activity and obesity prevention in children, adolescents and adults: a systematic review of prospective studies. Obes Rev. 2011 May;12(5):e119-29.

31. Church TS, Thomas DM, Tudor-Locke C, Katzmarzyk PT, Earnest CP, Rodarte RQ, Martin CK, Blair SN, Bouchard C. Trends over 5 decades in U.S. occupation-related physical activity and their associations with obesity. PLoS One. 2011;6(5):e19657.

32. Archer E, Lavie CJ, McDonald SM, Thomas DM, Hébert JR, Taverno Ross SE, McIver KL, Malina RM, Blair SN. Maternal inactivity: 45-year trends in mothers' use of time. Mayo Clin Proc. 2013 Dec;88(12):1368-77.

33. Archer E, Shook RP, Thomas DM, Church TS, Katzmarzyk PT, Hébert JR, McIver KL, Hand GA, Lavie CJ, Blair SN. 45-Year trends in women's use of time and household management energy expenditure. PLoS One. 2013;8(2):e56620. 
Table 1. Baseline characteristics in the total sample and by overall and abdominal obesity study groups in Taiwanese individuals from the MJ Cohort.

\begin{tabular}{|c|c|c|c|c|c|}
\hline & & \multicolumn{2}{|c|}{ Overall obesity* } & \multicolumn{2}{|c|}{ Abdominal obesity** } \\
\hline & All & No & Yes & No & Yes \\
\hline $\mathrm{N}$ & 113950 & 83567 & 24153 & 91700 & 12688 \\
\hline Women & 49.0 & 56.1 & 28.6 & 51.2 & 38.8 \\
\hline Age, y & $37.8 \pm 11.4$ & $36.8 \pm 11.1$ & $41.2 \pm 11.8$ & $36.5 \pm 10.6$ & $44.6 \pm 13.0$ \\
\hline \multicolumn{6}{|l|}{ Educational attainment, \% } \\
\hline Middle school or below & 14.0 & 11.8 & 21.4 & 10.8 & 30.6 \\
\hline High school & 20.2 & 20.6 & 18.9 & 20.5 & 18.3 \\
\hline Junior school & 24.6 & 25.6 & 21.7 & 25.7 & 18.9 \\
\hline College or above & 41.2 & 42.0 & 38.0 & 43.0 & 32.2 \\
\hline \multicolumn{6}{|l|}{ Marital status, \% } \\
\hline Single & 33.9 & 36.1 & 26.1 & 35.9 & 25.2 \\
\hline Married & 66.1 & 63.9 & 73.9 & 64.1 & 74.8 \\
\hline \multicolumn{6}{|l|}{ Smoking status, \% } \\
\hline Never & 73.9 & 77.1 & 64.2 & 75.4 & 66.2 \\
\hline Former & 6.0 & 5.1 & 8.6 & 5.6 & 8.2 \\
\hline Current & 20.1 & 17.8 & 27.2 & 19.0 & 25.6 \\
\hline \multicolumn{6}{|l|}{ Alcohol drinking, \% } \\
\hline Never & 83.1 & 85.3 & 76.2 & 84.5 & 76.5 \\
\hline Former & 2.1 & 1.8 & 3.0 & 1.9 & 3.2 \\
\hline Current & 14.8 & 12.9 & 20.8 & 13.7 & 20.3 \\
\hline Optimal meal patterns, $\%$ & 61.5 & 62.6 & 58.2 & 61.9 & 59.0 \\
\hline Body mass index, $\mathrm{kg} / \mathrm{m}^{2}$ & $22.7 \pm 3.4$ & $21.1 \pm 2.1$ & $27.7 \pm 2.4$ & $21.6 \pm 2.5$ & $28.4 \pm 3.0$ \\
\hline Overall obesity*, \% & 23.2 & 0 & 100 & 10.0 & 90.0 \\
\hline Hypertension, \% & 12.6 & 8.4 & 26.2 & 8.8 & 33.9 \\
\hline Hypercholesterolemia, \% & 9.9 & 8.0 & 15.9 & 8.2 & 18.2 \\
\hline Type 2 diabetes, $\%$ & 2.9 & 1.9 & 6.3 & 1.7 & 9.6 \\
\hline Physical activity, MET-h/week & $5.9 \pm 9.0$ & $5.6 \pm 8.8$ & $6.5 \pm 9.5$ & $5.8 \pm 9.1$ & $6.1 \pm 8.7$ \\
\hline
\end{tabular}

Values are mean $\pm \mathrm{SD}$ or $\%$. Overall obesity: body mass index $\geq 25 \mathrm{~kg} / \mathrm{m}^{2} . * *$ Abdominal obesity: waist circumference $\geq 90 \mathrm{~cm}$ in men and $\geq 80 \mathrm{~cm}$. 
Table 2. Association of changes in physical activity with the subsequent incidence and remission of overall and abdominal obesity.

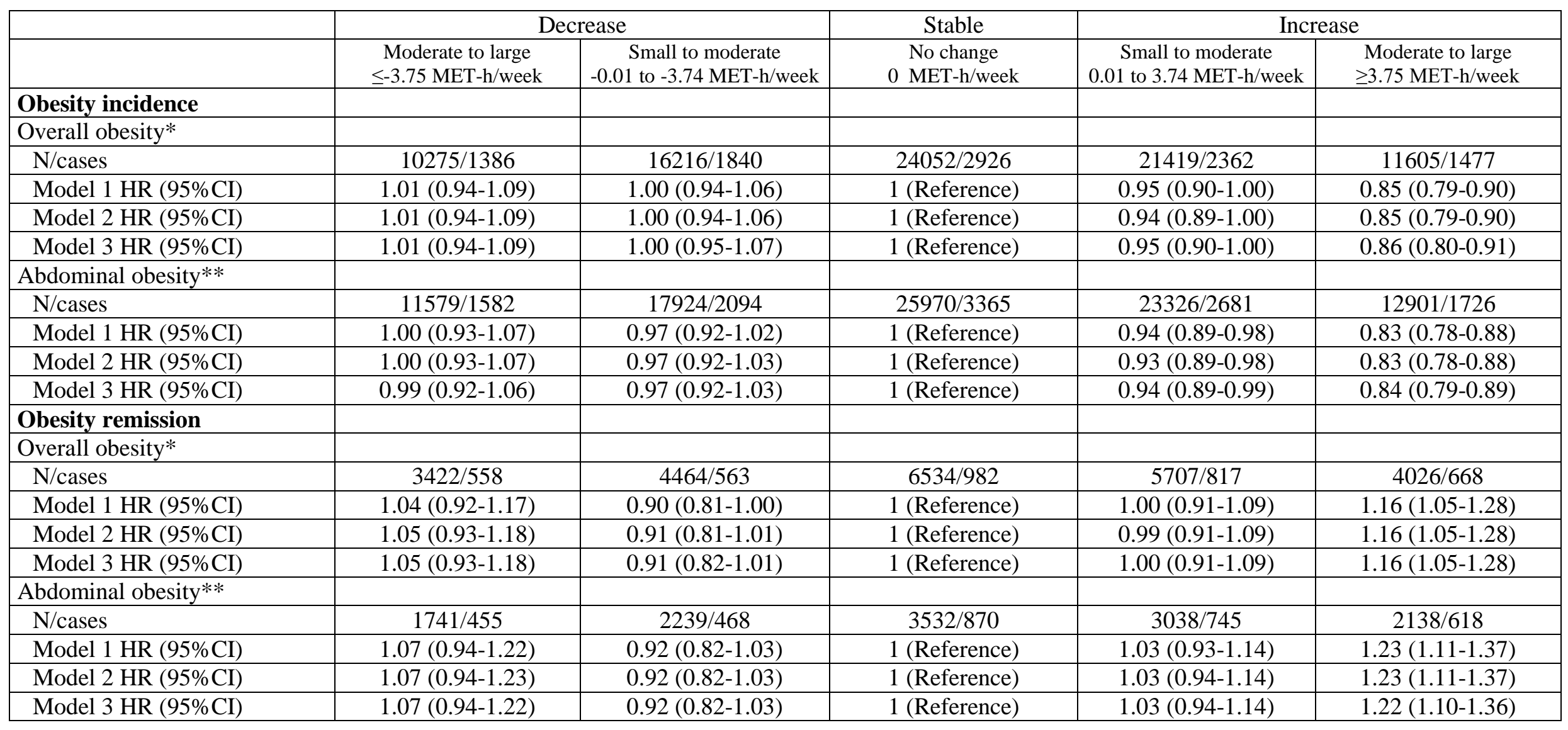

HR: Hazard ratio; CI: Confidence interval. *Overall obesity: body mass index $\geq 25 \mathrm{~kg} / \mathrm{m}^{2}$. **Abdominal obesity: waist circumference $\geq 90 \mathrm{~cm}$ in men and $\geq 80 \mathrm{~cm}$ in women.

Model 1 was adjusted for age, sex, year of baseline examination, educational attainment, marital status, body mass index, waist circumference in abdominal obesity analyses, and physical activity at baseline.

Model 2 was adjusted as Model 1 plus hypertension, hypercholesterolemia, and diabetes.

Model 3 was adjusted as model 2 plus baseline and changes in smoking, alcohol drinking, and meal patterns. 


\section{FIGURE LEGENDS}

Figure 1. Flowchart of participants in the Taiwan MJ Cohort. BMI: body mass index. WC: waist circumference. Overall obesity: $\mathrm{BMI} \geq 25 \mathrm{~kg} / \mathrm{m}^{2}$. Abdominal obesity: $\mathrm{WC} \geq 90 \mathrm{~cm}$ in men and $\geq 80 \mathrm{~cm}$ in women.

Figure 2. Incidence and remission of overall and abdominal obesity according to changes in physical activity, based on restricted cubic splines with knots at the $10^{\text {th }}, 50^{\text {th }}$, and $90^{\text {th }}$ percentile of the physical activity distribution. Solid lines indicate hazard ratios and dashed lines indicate $95 \%$ confidence intervals. Body mass index $\geq 25 \mathrm{~kg} / \mathrm{m}^{2}$ identified participants with overall obesity and waist circumference $\geq 90 \mathrm{~cm}$ in men and $\geq 80 \mathrm{~cm}$ in women identified participants with abdominal obesity. Analyses were adjusted for age, sex, year of baseline examination, educational attainment, marital status, body mass index, waist circumference in abdominal obesity analyses, physical activity at baseline examination, hypertension, hypercholesterolemia, diabetes, and baseline and changes in smoking, alcohol drinking, and meal patterns.

Figure 3. Incidence and remission of overall and abdominal obesity according to changes in physical activity categories. I bars represent $95 \%$ confidence intervals. Inactive: 0 MET-h/week. Insufficiently active: 0.01-7.49 MET-h/week. Active: $\geq 7.50 \mathrm{MET}-\mathrm{h} /$ week, equivalent to $150 \mathrm{~min} / \mathrm{wk}$ of moderate physical activity, $75 \mathrm{~min} / \mathrm{wk}$ at vigorous intensity, or a combination of both. Body mass index $\geq 25$ $\mathrm{kg} / \mathrm{m}^{2}$ identified participants with overall obesity and waist circumference $\geq 90 \mathrm{~cm}$ in men and $\geq 80 \mathrm{~cm}$ in women identified participants with abdominal obesity. Analyses were adjusted for age, sex, year of baseline examination, number of years between baseline and second examinations, educational attainment, marital status, body mass index, waist circumference in abdominal obesity analyses, hypertension, hypercholesterolemia, diabetes, and baseline and second examinations in smoking, alcohol drinking, and meal patterns. 